\title{
AQUISIÇÃO SEGMENTAL E PROSÓDICA NAS PRODUÇÕES VERBAIS DE CRIANÇAS PREMATURAS
}

\section{SEGMENTAL AND PROSODIC ACQUISITION IN VERBAL PRODUCTIONS OF PREMATURE CHILDREN}

\author{
Kyvia Fernanda Tenório da Silva ${ }^{1}$ \\ Luzia Miscow da Cruz Payão \\ Miguel José Alves de Oliveira Júnior ${ }^{3}$
}

\section{RESUMO}

$\mathrm{Na}$ aquisição de uma língua, a criança começa a realizar ajustes para produzir a língua-alvo (MATZENAUER; MIRANDA, 2010; MATZENAUER, 2015; PAYÃO; COSTA, 2016). Essa aquisição pode ser afetada pela idade gestacional e pelo peso. Este trabalho partiu da hipótese de que há entre os prematuros defasagens quanto à aquisição segmental consonantal, vocálica e prosódica, correlacionando a idade gestacional e o peso ao nascer. O objetivo da presente pesquisa foi analisar a aquisição segmental consonantal, vocálica e prosódica nas produções verbais de prematuros, considerando a idade gestacional e o peso ao nascer. Este estudo comparou a quantidade de segmentos consonantais e vocálicos e a quantidade de processos fonológicos entre os prematuros ao longo das seis coletas, tendo como referência a aquisição segmental e prosódica apontada pela literatura sobre desenvolvimento linguístico típico do PB. Os dados de fala espontânea de onze crianças, avaliadas entre 12 a 25 meses, provêm de um banco de dados longitudinal coletados entre 2014 e 2018. Os achados evidenciaram que os prematuros não produziram os segmentos plosivos e nasais da língua portuguesa até os 20 meses de idade corrigida, não distribuídos igualmente no preenchimento segmental quanto à tonicidade. A correção da idade não compensou a existência do atraso presente nos prematuros. A aquisição prosódica revelou-se defasada quanto ao preenchimento segmental das sílabas átonas e tônica, uma vez que a sílaba tônica se destacou em relação às átonas e os processos fonológicos de maior relevância foram a não realização de uma sílaba ou mais e a não realização de segmento no onset simples. Os prematuros estão em atraso por não preencherem os segmentos em todas as posições silábicas, podendo afetar o desempenho linguístico em idades posteriores.

PALAVRAS-CHAVE: Linguagem; Fala; Criança; Prematuro.

\begin{abstract}
When acquiring a language, the child begins to make adjustments to produce the target language (MATZENAUER; MIRANDA, 2010; MATZENAUER, 2015; PAYÃO; COSTA, 2016). This acquisition can be affected by gestational age and weight. This study started from the hypothesis that there are gaps between consonant, vocalic and premature prosodic segmental acquisitions, correlating gestational age with birth weight. The objective of this research was to analyze the consonant, vocal and prosodic segmental acquisition in the verbal productions of premature babies, considering the gestational age and birth weight. This study compared the number of consonant and vowel segments and the number of phonological processes in preterm infants over the six

\footnotetext{
${ }^{1}$ Doutoranda em Linguística pelo Programa de Pós-graduação em Linguística e Literatura da Universidade Federal de Alagoas. E-mail: kyviaftsilva@gmail.com

${ }^{2}$ Doutora em Linguística-Universidade Federal de Alagoas (UFAL). Professora Adjunto do curso de

Fonoaudiologia, compondo o Núcleo de Saúde Materno Infantil e do Adolescente - Centro de Ciências da Saúde da Universidade Estadual de Ciências da Saúde de Alagoas - UNCISAL. E-mail: luziapayao@uol.com.br

${ }^{3}$ Doutor em Linguística-Simon Fraser University (SFU). Professor Associado de Linguística na Universidade Federal de Alagoas e Bolsista de Produtividade em Pesquisa do CNPq - Nível 2. E-mail: miguel@fale.ufal.br
} 
collections, taking as a reference the segmental and prosodic acquisition indicated by the literature on typical development of BP. The spontaneous speech data of eleven children, assessed between 12 and 25 months, comes from a longitudinal database collected between 2014 and 2018. The findings showed that preterm infants did not produce the plosive and nasal segments of the Portuguese language until 20 months of corrected age, not uniformly distributed in the segment filling in terms of tonicity. Correction for age did not compensate for the delay present in preterm infants. The prosodic acquisition proved to be delayed in terms of segmental filling of unstressed and stressed syllables, since the stressed syllable stood out in relation to unstressed syllables and the most relevant phonological processes were not performing a syllable or more and not performing a thread in the simple onset. Premature babies are delayed because they do not fill segments in all syllabic positions, which can affect language performance at later ages.

KEYWORDS: Language; Speech; Child; Premature.

\section{INTRODUÇÃO}

A criança ao ser exposta a uma determinada língua, começa a realizar adaptações para produzir a língua-alvo, preenchendo as sílabas pertencentes à estrutura silábica da palavra, inicialmente com a tônica e, em seguida, com as átonas (MATZENAUER; MIRANDA, 2010; MATZENAUER, 2015; PAYÃO; COSTA, 2016).

No processo de aquisição, a criança passa por estágios no desenvolvimento linguístico, em que ocorre o incremento paulatino dos segmentos. Inicialmente, a criança percebe auditivamente as vogais, por apresentarem maior saliência perceptual. Isso colabora para a produção de estruturas silábicas compostas apenas por vogais, como onomatopeias e palavras monossílabas. A partir da percepção das diferenças fonológicas, a criança começa a adquirir todos segmentos da língua-alvo. Se acontecer a privação da exposição do input linguístico, haverá prejuízo na aquisição fonológica, favorecendo a ocorrência de uma fala ininteligível (WIETHAN; MOTA; MORAES, 2016).

A aquisição de uma língua pode ser afetada por questões relacionadas à idade gestacional e ao peso, porque, na primeira infância, sendo este período crítico para maturação cerebral, ocorre o desenvolvimento neurológico que em prematuros pode estar afetado. A literatura mostra que os volumes cerebrais podem ser um marcador útil dos resultados do desenvolvimento neurológico em prematuros moderados a tardios, tendendo estes a apresentar déficit cognitivo (JOHNSON et al., 2015; CHEONG et al., 2017a; CHEONG et al., 2017b), de linguagem (AGARWALL et al., 2018) e, em especial, na compreensão de vocabulário (RIBEIRO et al., 2016).

Esse desenvolvimento neurológico comprometido pelo déficit cognitivo também pode estar presente em bebês extremamente prematuros (ADAMS-CHAPMAN et al., 2018) e em crianças muito prematuras (PITTET-METRAILLER et al., 2019). Zerbeto, Cortelo e Filha (2015) revelaram que os prematuros exibem o pior desempenho linguístico, principalmente, relacionado à linguagem expressiva, tendo como fatores de risco o menor peso ao nascer e ao maior grau de prematuridade.

Boa parte das pesquisas que comparam o desempenho linguístico de prematuro com o de crianças típicas mostra que crianças pré-termo podem apresentar atraso neuropsicomotor e de linguagem (MONTEIRO-LUPERI et al., 2016; IONIO et al., 2016; RIBEIRO et al., 2016; SANCHES et al., 2019). Ainda são poucas as pesquisas no Português Brasileiro que tratam da aquisição e desenvolvimento linguístico de crianças prematuras.

Estudos sobre o desempenho linguístico de prematuros possuem implicações para profissões da área da saúde e educação, pois os prematuros tendem a apresentar atraso inicial na aquisição da língua. Dessa forma, esses profissionais e os pais precisam ser informados sobre o risco da defasagem linguística em prematuros, que pode ter repercussão ao longo da vida escolar. É importante haver o monitoramento do desenvolvimento global dos prematuros e, em especial, 
da aquisição da linguagem de forma longitudinal, podendo indicar a urgência do tratamento precoce.

A presente pesquisa teve como objetivo analisar a aquisição segmental consonantal, vocálica e prosódica nas produções verbais de crianças prematuras, considerando a idade gestacional e o peso ao nascer. O estudo partiu da hipótese de que há entre os prematuros deste estudo defasagens quanto à aquisição segmental consonantal, vocálica e prosódica, correlacionando com a idade gestacional e o peso ao nascer.

O estudo comparou a quantidade de segmentos consonantais e vocálicos e a quantidade de processos fonológicos entre os participantes da pesquisa ao longo das seis coletas, tendo como referência a aquisição segmental e prosódica apontada pela literatura sobre desenvolvimento linguístico típico do PB.

\section{Método}

O presente estudo se caracteriza como um estudo longitudinal e descritivo que tem por objetivo analisar a aquisição segmental consonantal, vocálica e prosódica nas produções verbais de crianças prematuras. O corpus de análise (fala espontânea de prematuros) provém de um banco de dados pertencente ao projeto de pesquisa de iniciação científica coordenado por uma professora pesquisadora de uma universidade pública estadual.

A pesquisa foi aprovada sob o CAAE no 78027717.0.0000.5011 - Comitê de Ética em Pesquisa - CEP da Universidade Estadual de Ciências da Saúde de Alagoas/UNCISAL e tem a anuência do Programa de Pós-graduação em Letras e Linguística - PPGLL- FALE/UFAL.

A seguir apresentaremos características gerais do referido banco de dados.

\subsection{Participantes de Pesquisa}

O banco de dados, constituído entre 2014 a 2018, é composto por transcrições fonéticas da fala espontânea de 12 participantes, sendo seis do gênero masculino e seis do gênero feminino, avaliados entre 12 e 25 meses de idade, que foram acompanhados em seis coletas. Para realização da presente pesquisa, foram usadas as transcrições fonéticas dos participantes que tivessem média igual ou superior a 24 palavras na sexta e última coleta. Dessa forma, 11 participantes foram incluídos por apresentar média igual ou superior a 24 palavras e 1 participante, S2, foi excluído por apresentar média inferior a 24 palavras, possibilitando à quantificação dos processos fonológicos, conforme expressam os dados da Tabela 1.

Tabela 1 - Totais e médias de palavras dos participantes ao longo das coletas

\begin{tabular}{lccccccc}
\hline Participantes & $\mathbf{1}^{\mathbf{a}}$ Coleta & $\mathbf{2}^{\mathbf{a}}$ Coleta & $\mathbf{3}^{\mathbf{a}}$ Coleta & $\mathbf{4}^{\mathbf{a}}$ Coleta & $\mathbf{5}^{\mathbf{a}}$ Coleta & $\mathbf{6}^{\mathbf{a}}$ Coleta & Média \\
\hline S1 & 35 & 44 & 61 & 53 & 61 & 81 & 55 \\
S3 & 6 & 19 & 33 & 52 & 47 & 72 & 38 \\
S4 & 8 & 19 & 41 & 26 & 48 & 106 & 41 \\
S5 & 11 & 18 & 36 & 26 & 55 & 45 & 31 \\
S6 & 16 & 22 & 27 & 31 & 12 & 36 & 24 \\
S7 & 21 & 36 & 57 & 49 & 46 & 57 & 44 \\
S8 & 19 & 37 & 33 & 95 & 118 & 123 & 70 \\
S9 & 14 & 35 & 19 & 52 & 17 & 50 & 31 \\
S10 & 20 & 32 & 64 & 46 & 86 & 107 & 59 \\
S11 & 13 & 33 & 72 & 64 & 89 & 101 & 62 \\
S12 & 18 & 21 & 64 & 84 & 66 & 65 & 53 \\
\hline \multicolumn{7}{r}{}
\end{tabular}


O Quadro 1, a seguir, traz a caracterização dos 11 participantes da pesquisa quanto aos dados gestacionais e sociodemográficos.

Quadro 1 - Caracterização dos participantes quanto aos dados gestacionais e sociodemográficos

\begin{tabular}{|c|c|c|c|c|c|c|}
\hline Participantes & Gênero & $\begin{array}{c}\text { Idade } \\
\text { Gestacional }\end{array}$ & $\begin{array}{c}\text { Peso } \\
\text { ao } \\
\text { nascer }\end{array}$ & $\begin{array}{c}\text { Escolaridade da } \\
\text { mãe }\end{array}$ & $\begin{array}{c}\text { Escolarização } \\
\text { da criança } \\
\text { Educação } \\
\text { Infantil }\end{array}$ & $\begin{array}{l}\text { Tempo total } \\
\text { de internação }\end{array}$ \\
\hline S1 & Feminino & 27 semanas & $905 \mathrm{~g}$ & Ensino Médio & Sim & 90 dias \\
\hline S3 & Masculino & 30 semanas & $1.135 \mathrm{~g}$ & Ensino Médio & Não & 60 dias \\
\hline S4 & Masculino & 30 semanas & $960 \mathrm{~g}$ & Ensino Médio & Não & 60 dias \\
\hline S5 & Feminino & 24 semanas & $785 \mathrm{~g}$ & $\begin{array}{c}\text { Ensino } \\
\text { Fundamental II }\end{array}$ & Não & 108 dias \\
\hline S6 & Masculino & 29 semanas & $1.445 \mathrm{~g}$ & Ensino Médio & Não & 45 dias \\
\hline S7 & Feminino & 32 semanas & $1.355 \mathrm{~g}$ & Ensino Médio & Não & 38 dias \\
\hline S8 & Feminino & 33 semanas & $1.835 \mathrm{~g}$ & Ensino Médio & Não & 16 dias \\
\hline S9 & Feminino & 35 semanas & $2.245 \mathrm{~g}$ & $\begin{array}{c}\text { Ensino } \\
\text { Fundamental II }\end{array}$ & Não & 7 dias \\
\hline S10 & Masculino & $\begin{array}{c}25 \text { semanas e } \\
4 \text { dias }\end{array}$ & $890 \mathrm{~g}$ & Ensino Médio & Não & 103 dias \\
\hline S11 & Feminino & $\begin{array}{c}30 \text { semanas e } \\
3 \text { dias }\end{array}$ & $1.190 \mathrm{~g}$ & Ensino Médio & Não & 47 dias \\
\hline S12 & Masculino & $\begin{array}{c}36 \text { semanas e } \\
2 \text { dias }\end{array}$ & $2.170 \mathrm{~g}$ & $\begin{array}{c}\text { Ensino } \\
\text { Fundamental II }\end{array}$ & Não & 5 dias \\
\hline
\end{tabular}

Fonte: Elaborado pela autora (2020).

\subsection{Descrição do banco de dados}

Para o desenvolvimento desta pesquisa, foi realizada a solicitação da dispensa do Termo de Consentimento Livre Esclarecido - TCLE - via Plataforma Brasil - CEP/UNCISAL, pela coordenadora do projeto de pesquisa original, responsável pela condução da pesquisa com as 12 crianças acompanhadas, para que o banco de dados pudesse ser consultado. Para mais informações sobre o banco de dados, consultar Silva e Payão (2020). Nesta pesquisa, os dados de fala de 11 participantes são confrontados com base na classificação pelo peso e pela idade gestacional, como mostra o Quadro 2, que podem favorecer as alterações de linguagem (ZERBETO; CORTELO; FILHA, 2015; SOARES; SILVA; ZUANETTI, 2017; FÉLIX; SANTOS; BENÍTEZ-BURRACO, 2017).

A OMS (2019) define pré-termo como a criança que nasceu com idade gestacional (IG) inferior a 37 semanas. A IG delimita o grau de prematuridade, sendo extremamente prematuro com menos de 28 semanas, muito prematuro de 28 a 32 semanas e prematuro moderado a tardio entre 32 e 37 semanas. Quanto ao peso, os prematuros podem ser classificados de baixo peso $(<2500 \mathrm{~g})$, de muito baixo peso $(<1500 \mathrm{~g})$ e de extremamente baixo peso $(<1000 \mathrm{~g})$ (ELEUTÉRIO et al., 2014).

Quadro 2 - Dados gestacionais dos participantes quanto à idade gestacional e ao peso

\begin{tabular}{|ll|}
\hline \multicolumn{1}{|c|}{ Classificação por Idade Gestacional e Peso } \\
\hline S1 & - Prematuros Extremos e Extremamente baixo peso \\
S4 & - Muito prematuro e Extremamente baixo peso \\
S5 & - Prematuros Extremos e Extremamente baixo peso \\
S10 - Prematuros Extremos e Extremamente baixo peso
\end{tabular}




\begin{tabular}{|ll|}
\hline S3 - Muito Prematuros e Muito baixo peso \\
S6 - Muito Prematuros e Muito baixo peso \\
S7 - Prematuros Moderado a Tardio e Muito baixo peso \\
S11 - Muito Prematuros e Muito baixo peso \\
\hline S8 - Prematuros Moderado a Tardio e Baixo peso \\
S9 - Prematuros Moderado a Tardio e Baixo peso \\
S12 - Prematuros Moderado a Tardio e Baixo peso \\
\hline
\end{tabular}

Fonte: Elaborado pela autora (2020).

Neste estudo, para confrontar os dados de fala dos participantes, ainda foi considerada a idade corrigida, sendo esta adaptada ao grau de prematuridade, considerando se tivesse nascido a termo, ou seja, com 40 semanas. A idade corrigida ajuda a compreender o desenvolvimento motor e de linguagem, pois podem ocorrer em momentos diferentes quando comparados a um bebê a termo (FORMIGA; VIEIRA; LINHARES, 2015).

Para calcular a idade corrigida do prematuro, é necessário subtrair a idade gestacional apresentada ao nascer das 40 semanas, que correspondem ao período completo de gestação de bebês a termo. Por exemplo, o participante S1 nasceu com 27 semanas, 40 semanas menos 27, o resultado é 13 semanas, que correspondem a 3 meses e 1 semana.

$\mathrm{Na}$ primeira coleta, S1 se encontrava com 1 ano, 9 meses e 22 dias de idade cronológica, subtraindo 3 meses e 7 dias, o resultado é 1 ano, 6 meses e 15 dias idade corrigida. Esse cálculo para a idade corrigida foi realizado para todos os participantes e convertido em meses. Assim, na primeira coleta S1 encontrava-se com 18 meses, como mostra o Quadro 3, a seguir. O cálculo da idade corrigida permite compreender como está o desempenho linguístico do participante comparado ao que a literatura aponta para criança com desenvolvimento típico naquela idade.

Quadro 3 - Dados dos participantes da pesquisa quanto à idade corrigida em meses apresentada em cada coleta

\begin{tabular}{|c|c|c|c|c|c|c|}
\hline \multicolumn{9}{|c|}{ Idade corrigida em meses } \\
\hline Participantes & $\mathbf{1}^{\mathbf{a}}$ Coleta & $\mathbf{2}^{\mathbf{a}}$ coleta & $\mathbf{3}^{\mathbf{a}}$ coleta & $\mathbf{4}^{\mathbf{a}}$ coleta & $\mathbf{5}^{\mathbf{a}}$ coleta & $\mathbf{6}^{\mathbf{a}}$ coleta \\
\hline S1 & $18 \mathrm{~m}$ & $19 \mathrm{~m}$ & $20 \mathrm{~m}$ & $21 \mathrm{~m}$ & $22 \mathrm{~m}$ & $23 \mathrm{~m}$ \\
\hline S3 & $18 \mathrm{~m}$ & $19 \mathrm{~m}$ & $20 \mathrm{~m}$ & $23 \mathrm{~m}$ & $24 \mathrm{~m}$ & $25 \mathrm{~m}$ \\
\hline S4 & $18 \mathrm{~m}$ & $19 \mathrm{~m}$ & $21 \mathrm{~m}$ & $23 \mathrm{~m}$ & $24 \mathrm{~m}$ & $25 \mathrm{~m}$ \\
\hline S5 & $13 \mathrm{~m}$ & $15 \mathrm{~m}$ & $17 \mathrm{~m}$ & $18 \mathrm{~m}$ & $20 \mathrm{~m}$ & $21 \mathrm{~m}$ \\
\hline S6 & $15 \mathrm{~m}$ & $17 \mathrm{~m}$ & $18 \mathrm{~m}$ & $20 \mathrm{~m}$ & $21 \mathrm{~m}$ & $22 \mathrm{~m}$ \\
\hline S7 & $16 \mathrm{~m}$ & $18 \mathrm{~m}$ & $19 \mathrm{~m}$ & $21 \mathrm{~m}$ & $22 \mathrm{~m}$ & $23 \mathrm{~m}$ \\
\hline S8 & $16 \mathrm{~m}$ & $18 \mathrm{~m}$ & $19 \mathrm{~m}$ & $21 \mathrm{~m}$ & $22 \mathrm{~m}$ & $23 \mathrm{~m}$ \\
\hline S9 & $16 \mathrm{~m}$ & $17 \mathrm{~m}$ & $20 \mathrm{~m}$ & $21 \mathrm{~m}$ & $23 \mathrm{~m}$ & $24 \mathrm{~m}$ \\
\hline S10 & $14 \mathrm{~m}$ & $15 \mathrm{~m}$ & $18 \mathrm{~m}$ & $20 \mathrm{~m}$ & $21 \mathrm{~m}$ & $22 \mathrm{~m}$ \\
\hline S11 & $15 \mathrm{~m}$ & $16 \mathrm{~m}$ & $18 \mathrm{~m}$ & $20 \mathrm{~m}$ & $22 \mathrm{~m}$ & $23 \mathrm{~m}$ \\
\hline S12 & $18 \mathrm{~m}$ & $20 \mathrm{~m}$ & $22 \mathrm{~m}$ & $23 \mathrm{~m}$ & $24 \mathrm{~m}$ & $25 \mathrm{~m}$ \\
\hline
\end{tabular}

Fonte: Elaborado pela autora (2020).

\subsection{Materiais}

Para realização da presente pesquisa, o banco de dados foi consultado, contendo a transcrição fonética das palavras, classificadas quanto ao número de sílabas e à acentuação e o inventário segmental das sílabas conforme a posição acentual nas palavras, demonstradas nos Quadros 4 e 5. Esses dois quadros foram inseridos com o intuito de exemplificar os procedimentos adotados no banco de dados que tinha o objetivo de quantificar, descrever e classificar as palavras produzidas por todos os participantes quanto ao número de sílabas e à tonicidade e quanto ao preenchimento segmental (consoante $1-\mathrm{C} 1$, consoante $2-\mathrm{C} 2 \mathrm{em}$ posição de onset - consoantes de início da sílaba; vogal - V, glide como as vogais em núcleo de sílaba, consoante 3 - C3, representando a coda silábica, a consoante em final da sílaba) correspondente às sílabas tônica, prétônica e pós-tônica. 
Quadro 4 -Classificação das palavras produzidas quanto ao $\mathrm{n}^{\circ}$ de sílabas e à acentuação

\begin{tabular}{|c|c|c|c|c|c|c|}
\hline \multirow{2}{*}{ Onomatopeia } & \multicolumn{2}{|c|}{ Monossílabos } & \multirow{2}{*}{$\begin{array}{c}\text { Dissílabas } \\
\text { Oxítonas }\end{array}$} & \multirow{2}{*}{$\begin{array}{l}\text { Dissílabas } \\
\text { Paroxítonas }\end{array}$} & \multirow{2}{*}{$\begin{array}{c}\text { Tris. e Poli } \\
\text { Paroxít. Oxítona }\end{array}$} & \multirow{2}{*}{$\begin{array}{c}\text { Tris. } \\
\text { Propar. }\end{array}$} \\
\hline & Átonos & Tônicos & & & & \\
\hline $\begin{array}{c}\text { [aw.aw] } \\
\text { Auau } \\
\text { [ko.'ko] } \\
\text { Onomato } \\
\text { galinha }\end{array}$ & $\begin{array}{l}{[\mathrm{a}]} \\
\mathrm{a}\end{array}$ & $\begin{array}{l}{[\text { nãw] }} \\
\text { Não } \\
{[' \varepsilon]} \\
\text { E } \\
{[' p \varepsilon]} \\
\text { Pé } \\
\text { [tẽ] } \\
\text { Tem } \\
\text { [di3] } \\
\text { Diz } \\
\text { [mãw] } \\
\text { Mão } \\
\text { ['ke] } \\
\text { Quer }\end{array}$ & $\begin{array}{l}\text { [a.'i] } \\
\text { Aí } \\
\text { [nẽ.'nẽ] } \\
\text { Neném } \\
\text { [bo.'bo] } \\
\text { Vovô } \\
\text { [mã.'mai] } \\
\text { Mamãe } \\
\text { [ma.'ma] } \\
\text { Mamar } \\
\text { [i.'ãw] } \\
\text { Leão } \\
\text { ['j’] } \\
\text { Fechou } \\
\text { [a.'biu] } \\
\text { Abriu } \\
\text { ['ki] } \\
\text { Aqui } \\
\text { [pa.'paj] } \\
\text { papai }\end{array}$ & $\begin{array}{l}\text { ['da.to] } \\
\text { Gato } \\
\text { ['bũ.bũ] } \\
\text { Bumbum } \\
\text { ['do.ew] } \\
\text { Doeu } \\
\text { [‘bãw] } \\
\text { Sabão } \\
\text { ['do] } \\
\text { Guardou } \\
\text { ['ka] } \\
\text { Está } \\
\text { ['pej.to] } \\
\text { Peito } \\
\text { ['ti.a] } \\
\text { Tia }\end{array}$ & $\begin{array}{l}\text { [mĩ.'nĩ.na] } \\
\text { Menina } \\
\text { [po.'po.ka] } \\
\text { Pipoca } \\
\text { [bi.'toj.tu] } \\
\text { Biscoito } \\
\text { [pa.'pa.pu] } \\
\text { Sapato } \\
\text { [nã.'nã.na] } \\
\text { Banana } \\
\text { [na.'nõ.ni] } \\
\text { Danone } \\
\text { [bi.'bi] } \\
\text { Bianca }\end{array}$ & \\
\hline Totais: 2 & & 1 & 10 & 9 & $7=35$ pala & \\
\hline
\end{tabular}

Quadro 5 - Inventário segmental das sílabas conforme a posição acentual nas palavras

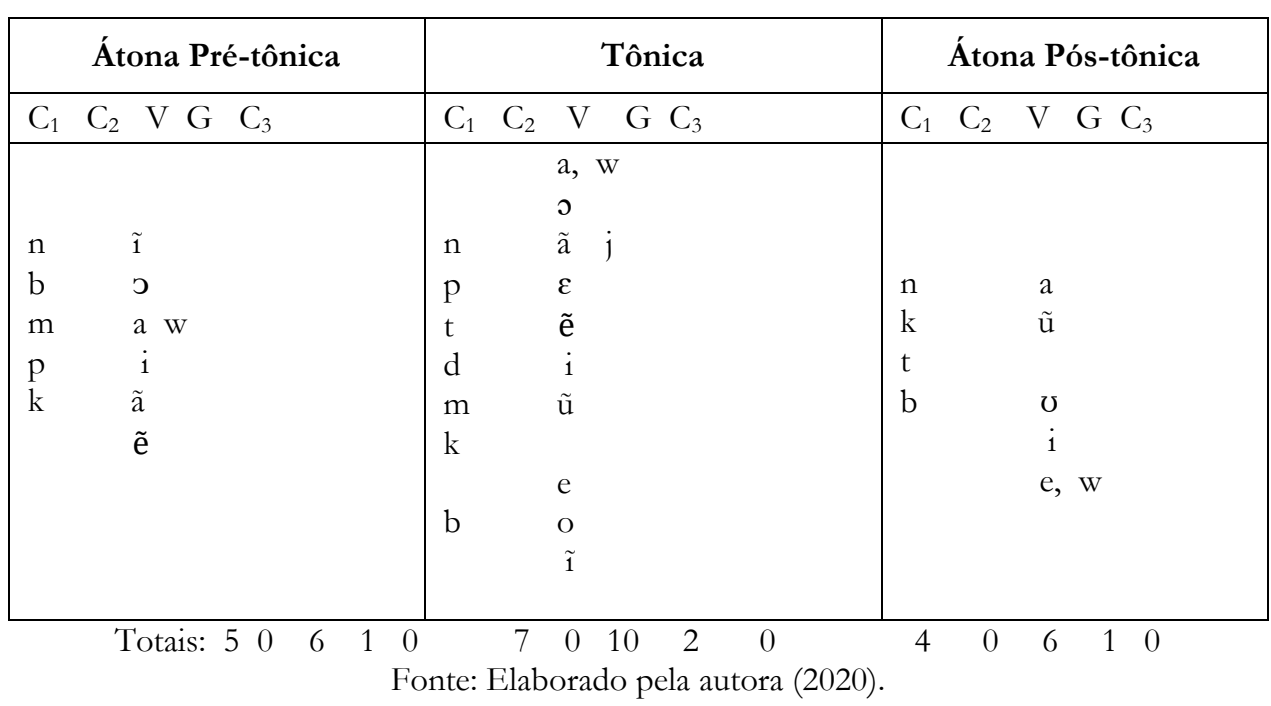

Tomando-se por base essa consulta, foi elaborado uma tabela ${ }^{4}$ no Excel com a quantidade dos segmentos consonantais e vocálicos, a tonicidade, os participantes, o gênero, o peso, a idade corrigida, o segmento (consonantal/ vocálico), a idade gestacional e a coleta.

Para analisar os segmentos consonantais, foi elaborado outra tabela ${ }^{4}$ com a descrição dos traços distintivos $(0 \mathrm{TM}=$ zero traço marcado, $1 \mathrm{TM}=$ um traço marcado, $2 \mathrm{TM}=$ dois traços

\footnotetext{
${ }^{4}$ Tabelas disponíveis no site https://github.com/KyviaTFSilva/Dados-disserta-o.git
} 
marcados), as classes segmentais $(\mathrm{pl}=$ plosiva, $\mathrm{ns}=$ nasal, $\mathrm{fr}=$ fricativa, $\mathrm{lq}=$ líquida $)$, a tonicidade, os participantes, o gênero, o peso, a idade corrigida, a idade gestacional e o tipo de segmento. Para as classes foram usados códigos, como ausente, presente, contém, não contém, tem e não tem e 0 e 1 para que o software $R$ pudesse fazer a análise da presença e ausência das classes segmentais.

Com base na consulta dos quadros de transcrições fonéticas das palavras, as estratégias de reparo, ou seja, os processos fonológicos empregados, foram identificadas no nível segmental como dessonorização de obstruentes, sonorização de obstruentes, anteriorização de velar, posteriorização para palatal, semivocalização de líquidas, substituição de líquida, não realização do segmento em onset simples, palatalização, plosivização, assimilação/harmonia consonantal e/ou vocálica; e no nível silábico, como a não realização do segundo membro de um onset complexo, a não realização de coda e a não realização de uma sílaba ou mais.

Após a identificação dos processos fonológicos, foi construído uma outra tabela ${ }^{4}$, contendo participantes, gênero, peso, idade, os processos fonológicos, quantidade de processos, idade gestacional, coletas, tipo (segmental ou silábico) e processos ( $n=$ não, $s=\operatorname{sim}$ ).

Considerando a hipótese quanto às diferentes defasagens nos pré-termo correlacionando à idade gestacional e ao peso, aplicou-se modelo estatístico de regressão multinível, que possibilita controlar variáveis "participantes" e "coletas". Quando esse controle não é realizado, "participantes" e "coletas" poderiam superestimar o efeito, ou seja, mostrar relevância entre um grupo de participantes ou em determinadas coletas em detrimento de outros.

Para análise estatística dos dados, utilizou-se o software $\mathrm{R}$ para analisar os dados, tendo quantidade (segmentos ou processos fonológicos) como variável dependente e peso e idade gestacional como efeitos fixos (variáveis independentes), controlando as coletas e os participantes, resultados significativos com $\mathrm{p} \leq 0,05$. Além disso, aplicou-se modelo estatístico de regressão multinível para analisar os dados, tendo quantidade (segmentos ou traços ou processos fonológicos) como variável dependendo e idade corrigida em meses como variável independente, controlando as coletas e os participantes, resultados significativos $\mathrm{p} \leq 0,05$.

\section{Resultados e Discussão}

À medida que ocorre o aumento da idade (13 meses a 25 meses), a quantidade dos segmentos cresce (3 para 12), como mostra a Figura 1, resultante do modelo estatístico de regressão linear multinível, tendo a quantidade de segmentos como variável dependente e idade em cada coleta como variável independente. Os resultados contêm interceptos aleatórios por participantes e por coletas, ou seja, foram controlados para evitar superestimar o efeito. Os achados corroboram a literatura ao expor que a aquisição segmental ocorre paulatinamente (LAMPRECHT et al., 2004; MIRANDA; MATZENAUER, 2009; MOTA, 1996).

Figura 1- Índice de quantidade de segmentos por idade corrigida em meses idade effect plot

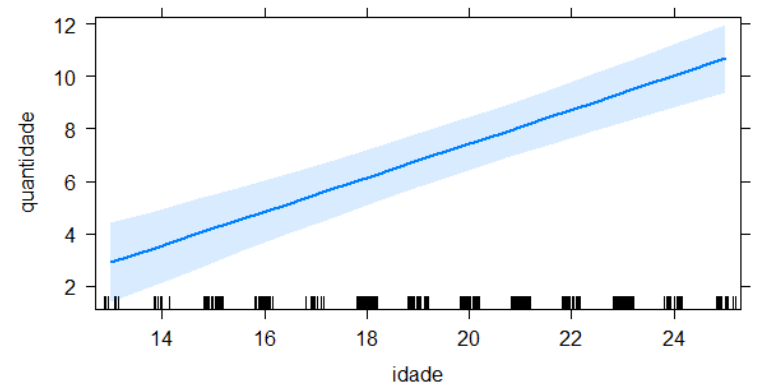

Fonte: Elaborada pela autora (2020).

$\mathrm{P}<0.001$, tendo referência de significância $\mathrm{p} \leq 0,05$. 
Ao aplicar o modelo de regressão multinível, tendo as classes plosivas, nasais, fricativas, líquidas e tonicidade como variável dependente e a idade corrigida em cada coleta como variável independente, contendo interceptos aleatórios por participantes, os dados mostraram significância, revelando o aumento da quantidade de segmentos em todas as classes segmentais com o acréscimo da idade, como demonstra a Figura 2.

Figura 2 - Índice da ocorrência das classes nasais, plosivas, fricativas e líquidas em relação à idade corrigida em meses
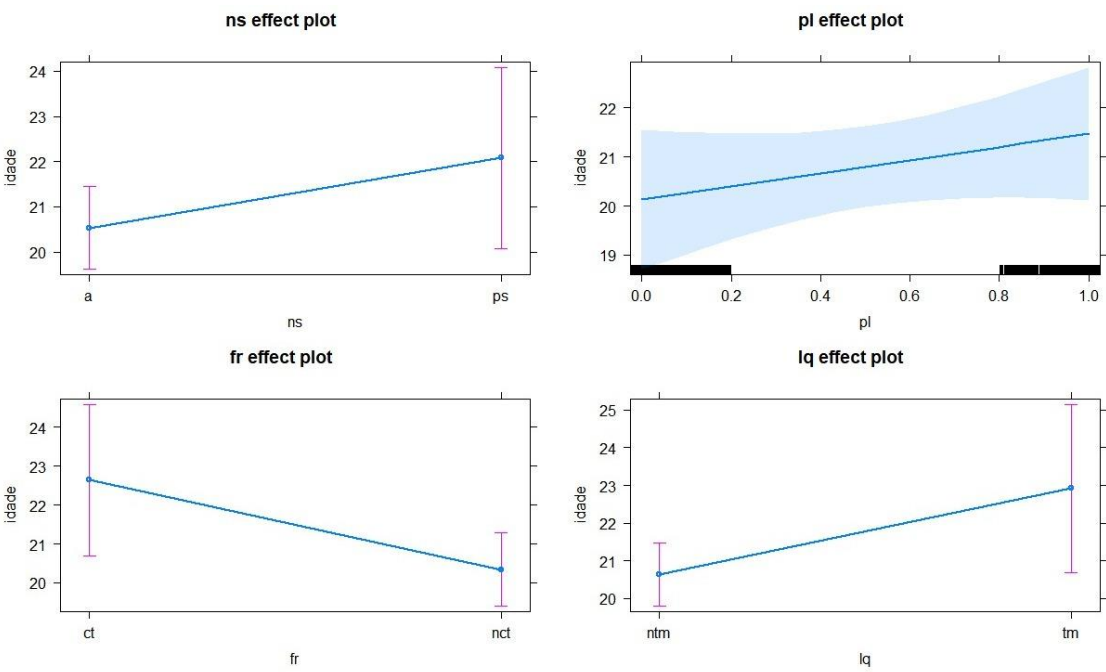

Fonte: Elaborada pela autora (2020).

$\mathrm{P}<0.001$, referência de significância $\mathrm{p} \leq 0,05$.

Legenda: $\mathrm{pl}=$ plosiva, $\mathrm{ns}=$ nasal, $\mathrm{lq}=$ líquida, $\mathrm{fr}=$ fricativa, $\mathrm{a}=$ ausente, $\mathrm{os}=$ presente, $\mathrm{ntm}=$ não tem $, \mathrm{tm}=\mathrm{tem}, \mathrm{ct}$ $=$ contém, nct $=$ não contém

Putnick, Bornstein, Eryigit-Madzwamuse, Wolke (2017) comentam que aos 20 meses de idade corrigida é esperado que os pré-termo tenham desempenho semelhante aos do termo. Há idade esperada para aquisição de cada classe de segmentos: plosivas e nasais até 20 meses, fricativas até 34 meses e líquidas, 48 meses (FREITAS, 2004; MEZZOMO E RIBAS, 2004; OLIVEIRA, 2004).

Os dados da presente pesquisa, entretanto, não evidenciam que as crianças produziram todos os segmentos da língua portuguesa até os 20 meses de idade corrigida, como preconiza a literatura. A correção da idade que serve para entender o desenvolvimento de linguagem quando comparados a um bebê a termo (FORMIGA, VIEIRA, LINHARES, 2015), mas essa correção não compensou a existência do atraso presente nos prematuros.

Ao analisar os traços distintivos dos segmentos consonantais a partir da idade corrigida apresentada pelos participantes, houve significância, como se observa na Figura 3. Segundo Mota (1996), os traços distintivos possuem níveis de complexidade, sendo os fonemas $/ \mathrm{p} / \mathrm{,} / \mathrm{t} / \mathrm{,} / \mathrm{m} / \mathrm{e}$ $/ \mathrm{n} /$ de menor complexidade e os primeiros a surgirem no inventário fonológico.

Os dados da pesquisa mostram a presença dos fonemas de menor complexidade pertencente a classe das plosivas e das nasais surgem antes dos 20 meses e que os segmentos de maior complexidade, aqueles que exigem a combinação de dois traços para produção dos segmentos que irão compor mais tardiamente o inventário aos 25 meses.

Os traços distintivos possibilitam que os segmentos sejam reunidos em conjuntos a partir das características articulatória e acústica. Apesar dos prematuros produzirem segmentos que possuem dois traços /g/ [dorsal, +voz], / $/ \mathrm{g} / \mathrm{H}$ [+contínuo, -anterior], /r/[+aproximante, + contínuo] e $/ K /$ [+aproximante, -anterior], isso não significa que tenham adquiridos todos os traços de contraste do português brasileiro, pois os participantes não preenchem os segmentos aos 
25 meses em todas as posições silábicas como também não possuem todos fonemas das classes segmentais (FREITAS, 2004; MEZZOMO E RIBAS, 2004; OLIVEIRA, 2004). À medida que os participantes vão estabelecendo novos contrastes na tentativa de produzir o sistema-alvo, ocorre a inclusão de traços que, a priori, eram limitados (CLEMENTS, 2005), justificando a disposição do gráfico na Figura 3.

Figura 3- Índice da média da idade corrigida em meses apresentada em cada coleta em relação aos traços distintivos

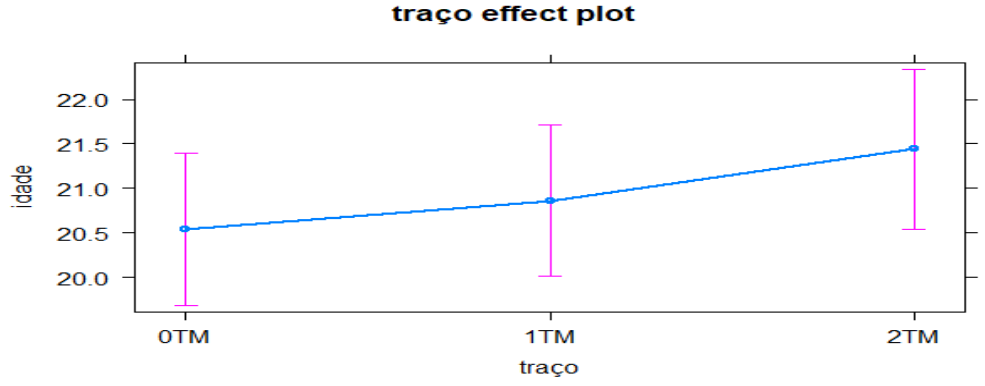

Fonte: Elaborada pela autora (2020).

$\mathrm{P}<0.001$, referência de significância $\mathrm{p} \leq 0,05$.

Legenda: $0 \mathrm{TM}=$ zero traço marcado, $1 \mathrm{TM}=$ um traço marcado, $2 \mathrm{TM}=$ dois traços marcados

É esperado que à medida que a criança adquira os segmentos, a produção de processos fonológicos diminua. Entretanto, os dados sinalizaram que a quantidade de processos fonológicos cresceu com aumento da idade até 25 meses, como indica a Figura 4 abaixo.

Figura 4- Índice da quantidade de processos fonológicos em relação à idade corrigida em meses apresentada em cada coleta

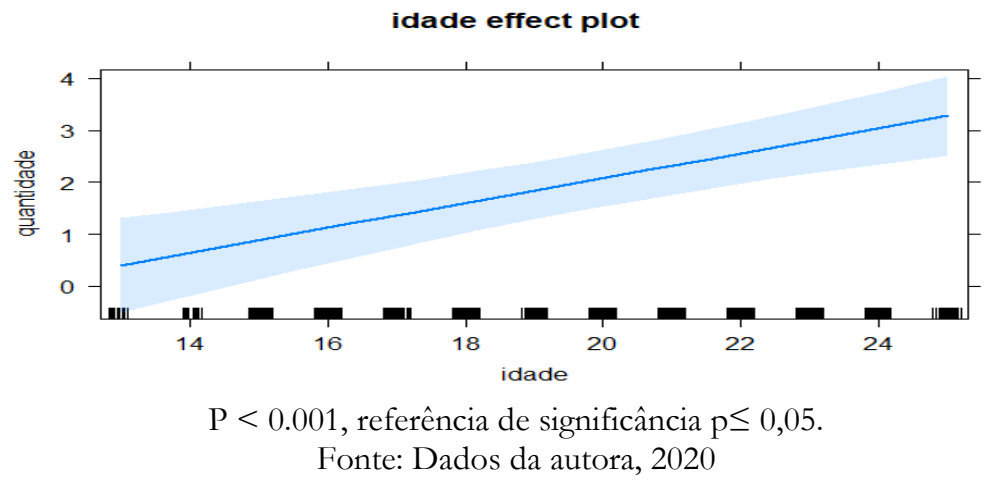

O crescimento do número de processos fonológicos revela que a criança está começando a dominar as regras fonológicas pertencentes ao sistema linguístico que está sendo exposta. A criança, além de perceber auditivamente o contraste entre os fonemas, preenche os segmentos nas sequências das estruturas silábicas que constituem a palavra-alvo como mostram os dados: S1 (21 meses) [pa.'pa.lv] cavalo, S3 (23 meses) [ka.'̂̃. ] carrinho, S4 (23 meses) [pa.'pa.to] sapato, S5 (18 meses) [pe.'si. no] peixinho, S6 (20 meses)['a.oj] arroz, S7 (21 meses)[de.'de.da] cerveja, S8 ( 21 meses)[di.'ej.to] direito, S9 (21 meses) [ta. 'dĩ.na] casinha, S10 (20 meses) [si.'si.a] Sofia, S11 (20 meses) [a.ma.'je.jo] amarelo e S12 (22 meses) [E.'fã.tI] elefante.

A ocorrência crescente dos processos fonológicos com o aumento da idade revela que as crianças tendem a fazer omissões e substituições de segmentos ou sílabas como estratégias para conseguir alcançar a palavra-alvo do adulto. Essas omissões e substituições ocorrem devido ao inventário segmental no processo de aquisição ser inicialmente limitado, mas também revelam que 
o processo de aquisição fonológica ainda está em andamento e que os prematuros estão organizando o nível fonético no nível fonológico para alcançar o sistema-alvo

A literatura aponta que existe uma idade esperada para que os processos fonológicos sejam superados. Segundo Wertzner (1992), a idade de eliminação dos processos fonológicos ocorre entre 2 anos e 6 meses e 7 anos. Os prematuros da presente pesquisa foram avaliados até 2 anos e 1 mês, o que justifica a permanência da ocorrência produtiva dos processos fonológicos por não possuírem idade para eliminá-los e por ainda estar em aquisição fonológica.

Lamprecht et al. (2004) mostram que os processos fonológicos podem ocorrer no nível segmental e silábico. Pode-se observar nos resultados os seguintes processos fonológicos no nível silábico: não realização de coda com $\mathrm{p}=0.003$, não realização de uma sílaba ou mais $-\mathrm{p}<0.0001$, não realização do segundo membro de um onset complexo $-\mathrm{p}=0.03$. No nível segmental, os que apresentaram relevância estatística foram: plosivização de fricativa $(p=0.02)$ e a não realização do segmento em onset simples - $\mathrm{p}<0.0001$, segue a Figura 5 abaixo.

Figura 5- Índice da quantidade dos processos fonológicos em função da idade corrigida em meses em cada coleta

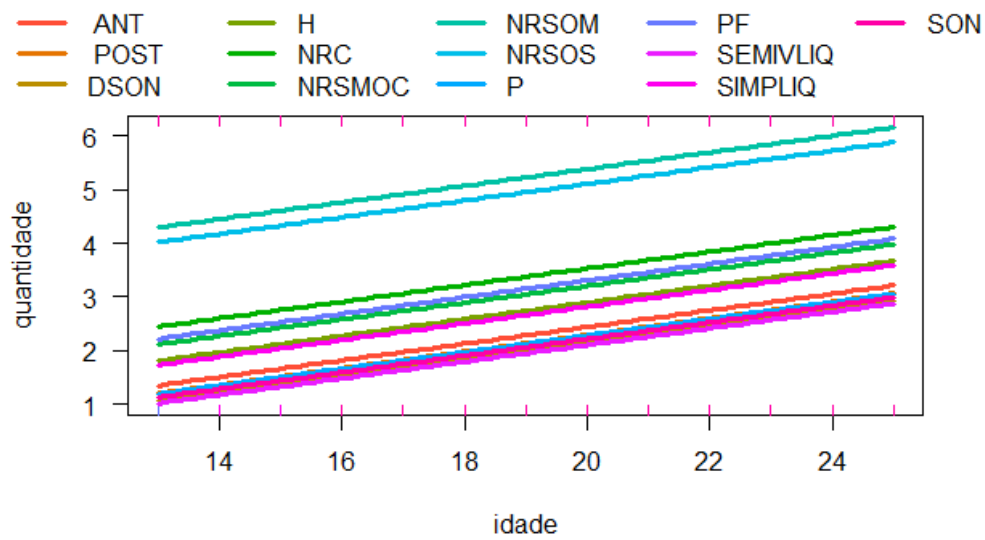

$\mathrm{P}<0.0001$, referência de significância $\mathrm{p} \leq 0,05$.

Legenda: ant $=$ anteriorização velar, post $=$ posteriorização para palatal, dson $=$ dessonorização, $\mathrm{h}=$ harmonia, nrc $=$ não realização de coda, nrsmoc $=$ não realização do segundo membro do onset complexo, nrsom = não realização de uma sílaba ou mais, nrsos = não realização do segmento em onset simples, $\mathrm{p}=$ palatalização, $\mathrm{pf}=$ plosivização de fricativa, semivliq = semivocalização de líquida, simpliq = simplificação de líquida, son= sonorização.

Fonte: Dados da autora, 2020.

Os segmentos tendem a se diferenciar quanto à classe de sons, à posição na sílaba e na palavra, sendo norteados pela aquisição prosódica. No que se refere à tonicidade, os segmentos ocupam inicialmente a sílaba tônica, responsável por desencadear os preenchimentos das demais sílabas átonas (PAYÃO, 2010; MATZENAUER, 2015; PAYÃO; COSTA, 2016).

Constatou-se em nossos dados que a sílaba tônica teve destaque significativo, sendo mais produzida $(\mathrm{p}<0.0001)$ em relação às sílabas pré-tônica e pós-tônica. A sílaba pós-tônica, tanto para os segmentos consonantais quanto para os segmentos vocálicos, apresentou relevância estatística $(p=0.002)$, sendo a segunda sílaba em relação à tonicidade com maior produção, como se observa na Figura 6 abaixo. 
Figura 6- Índice de quantidade de segmentos consonantais e vocálicos por tonicidade tonicidade effect plot

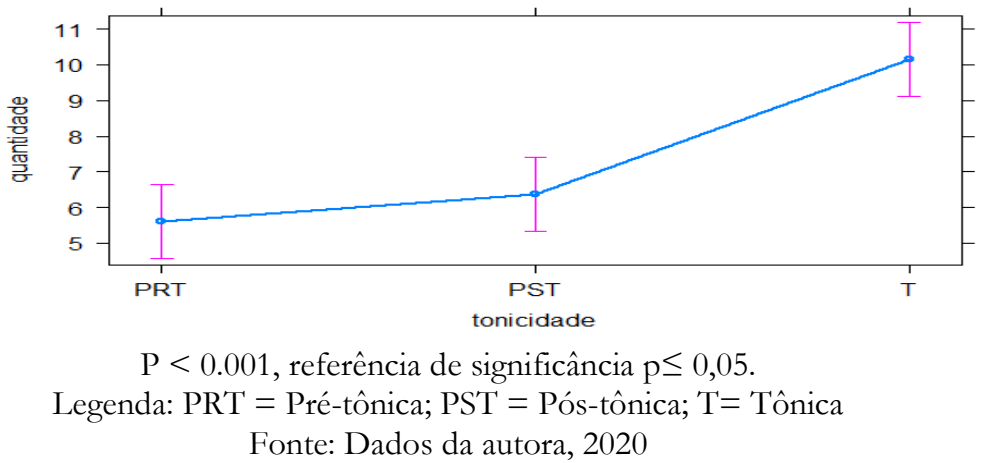

Segundo Lamprecht et al. (2004), aos 20 meses as crianças a termo deveriam ter adquirido as classes plosivas e nasais em onset absoluto e medial. Os prematuros deste estudo, aos 20 meses, não realizaram o preenchimento desses segmentos nas posições átonas e tônicas igualmente, indicando a presença do atraso.

Há presença do atraso na aquisição e no desenvolvimento fonológico em prematuros quanto à aquisição prosódica, no que se refere ao preenchimento segmental das posições silábicas - átonas e tônica. Os pré-termo faziam, inicialmente, a segmentação da sílaba tônica, sendo esta responsável pela segmentação silábica da palavra, em razão da proeminência entonacional do enunciado do input. A presença do atraso em prematuros persiste mesmo após os 24 meses e nem a correção da idade faz compensar (IONIO et al., 2016; MONTEIRO-LUPERI et al., 2016).

A ocorrência dessa segmentação feita pelos participantes é alta, uma vez que o processo fonológico de não realização de uma sílaba ou mais da palavra perdurou até a última coleta. Além disso, observou-se, nas produções da sexta e última coleta, que os participantes ainda estruturavam as palavras trissílabas ou polissílabas reduzindo-as ao pé métrico trocaico em alguns casos: $\mathrm{S} 1(23 \mathrm{~m})$ ['vi.ãw] avião, S3 (25m) ['tã.ma] Tamara, S4 (25m) ['kJ.da] acorda, S5 (20m) ['a.vo] árvore, S6 (22m) ['a.vi] árvore, S7 (23m) ['a.ta] barata, S8 (23m) [ma.'je.a] amarela, S9 (24m) ['sã.tI] elefante, S10 (22m) ['i.ãw] avião, S11 (23m) ['ãw] macarrão e S12 (24m) [fa.'ãw] macarrão.

Vários trabalhos mostraram que a idade gestacional é um dos fatores de risco para a defasagem no desempenho linguístico (ZERBETO; CORTELO; FILHA, 2015; SOARES; SILVA; ZUANETTI, 2017; FÉLIX; SANTOS; BENÍTEZ-BURRACO, 2017). Entretanto, os resultados desta pesquisa evidenciaram que idade gestacional não foi relevante com relação à quantidade de segmentos consonantais e vocálicos durante as seis coletas, apresentando $\mathrm{p}=0.2$ para prematuros extremos e $\mathrm{p}=0.5$ para prematuros moderados a tardio, como mostra a Figura 7 abaixo.

Figura 7- Índice de quantidade de segmentos consonantais e vocálicos em relação à idade gestacional ig effect plot

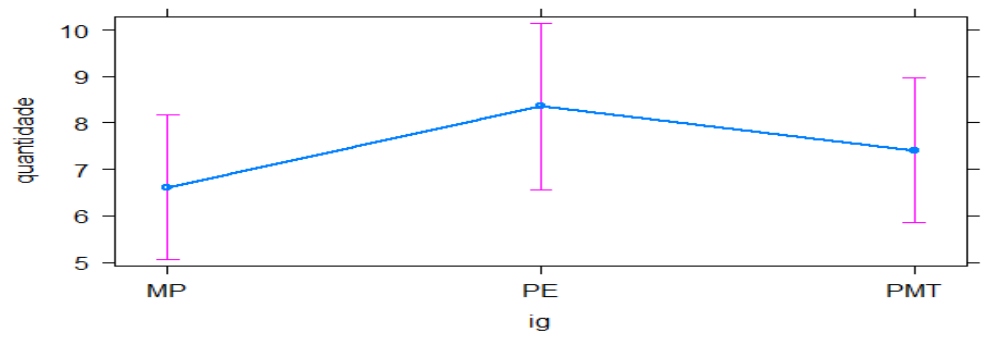

Legenda: Extremamente prematuros $(\mathrm{PE})<28$ semanas; Muito prematuros (MP) entre 28 e 32 semanas; Prematuros; Moderados a tardio (PMT) entre 32 e 36 semanas Fonte: Dados da autora, 2020. 
Além da idade gestacional não ter relevância para quantidade de segmentos, também não houve significância para quantidade em relação aos processos fonológicos, tendo $p=0.3$ para prematuros extremos e $\mathrm{p}=0.3$ para prematuros moderados a tardio, como mostra a Figura 8 .

Figura 8- Índice de quantidade de processos fonológicos em relação à idade gestacional ig effect plot

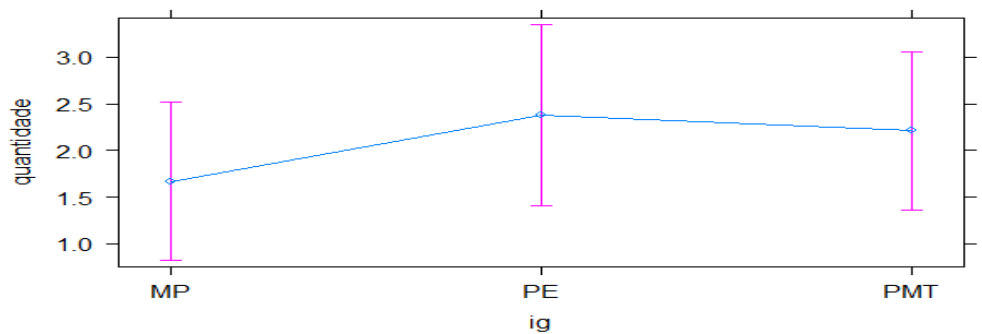

Legenda: Extremamente prematuros $(\mathrm{PE})<28$ semanas; Muito prematuros (MP) entre 28 e 32 semanas; Prematuros; Moderados a tardio (PMT) entre 32 e 36 semanas Fonte: Dados da autora, 2020

Outros estudos destacaram que o peso ao nascer é uma variável associada à prematuridade como responsável pelo atraso linguístico (HOLLANDERS et al., 2019; ZAGO et al., 2017). No entanto, os atuais dados indicaram que a quantidade de segmentos consonantais e vocálicos não foi significativa, tendo $\mathrm{p}=0.9$ para participantes com extremo baixo peso $(\mathrm{EBP})$ e $\mathrm{p}=0.8$ para participantes com muito baixo peso (MBP), segue a Figura 9.

Figura 9- Índice da quantidade de segmentos consonantais e vocálicos em relação ao peso ao nascer

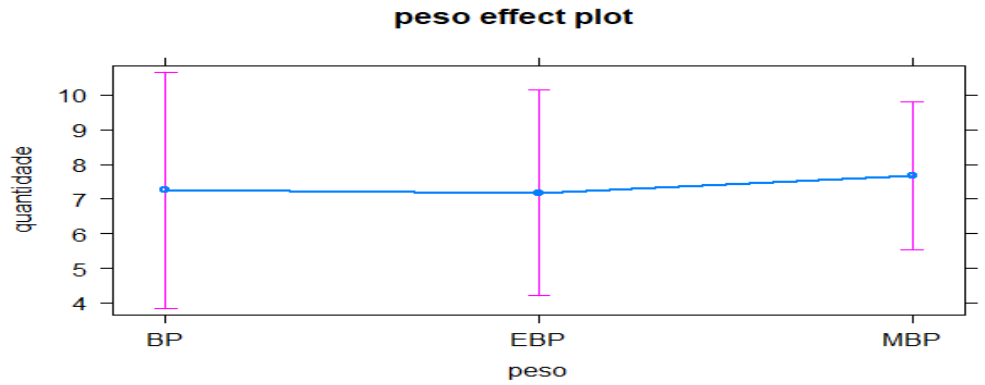

Legenda: Baixo peso $=$ BP; Extremo baixo peso $=$ EBP; Muito baixo peso $=$ MBP Fonte: Dados da autora, 2020.

Além de não ser relevante para segmentos, o peso ao nascer também não mostrou significância estatística entre os participantes quanto à quantidade de processos fonológicos, sendo $\mathrm{p}=0.7$ para extremo baixo peso e $\mathrm{p}=0.2$ para muito baixo peso, segue a Figura 10 .

Figura 10- Índice da quantidade de processos fonológicos em relação ao peso ao nascer peso effect plot

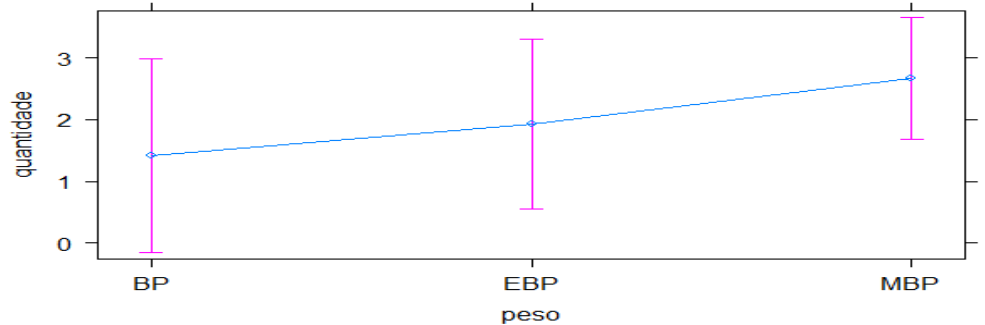

Legenda: Baixo peso = BP; Extremo baixo peso $=\mathrm{EBP} ;$ Muito baixo peso $=\mathrm{MBP}$ Fonte: Dados da autora, 2020. 
Os dados desta pesquisa revelaram que, entre os prematuros, quanto à idade gestacional e ao peso, não apresentaram diferenças. Dessa forma, qualquer grau de prematuridade e estratificação de peso poderá acarretar comprometimento na aquisição segmental e prosódica.

\section{CONSIDERAÇÕES FINAIS}

A aquisição prosódica entre os prematuros de diferentes graus de prematuridade e estratificação de peso revelou-se defasada no preenchimento segmental quanto à tonicidade, pois a sílaba tônica se destacou por apresentar maior quantidade de segmentos em detrimento das átonas, indicando a presença do atraso.

Além disso, aos 20 meses, os prematuros não preencheram os segmentos plosivos e nasais em onset absoluto e medial, favorecendo para produção de processos fonológicos que tiveram maior relevância: não realização de uma sílaba ou mais e não realização de segmento no onset simples.

De acordo com a literatura, os processos fonológicos diminuem com o aumento da idade, mas os dados da presente pesquisa revelaram que ocorre aumento dos processos fonológicos com o aumento da idade, pois os participantes ainda estavam em processo de aquisição fonológica, justificando as omissões e substituições nas produções verbais dos prematuros. Os prematuros, ao perceber auditivamente as distinções dos segmentos, empregavam esses segmentos disponíveis em seu inventário fonológico nas posições silábicas, manifestando as diferentes produções fonéticas para alcançar uma palavra-alvo.

Ademais, os prematuros não possuíam idade de superar os processos fonológicos, tendo idade média de 23 meses, por causa da imaturidade neuromuscular para produzir os segmentos da língua-alvo.

Observou-se também que o sistema fonológico dos prematuros é regido pelo princípio da evitação dos traços marcados, uma vez que há ocorrência maior de plosivas do que fricativas. A presença desse atraso inicial poderá afetar o desempenho linguístico futuramente.

Além disso, os dados de fala espontânea dos 11 participantes foram confrontados com base nas variáveis IG e peso ao nascer. Ao comparar os dados quanto à quantidade de segmentos consonantais e vocálicos e à quantidade de processos fonológicos dos participantes extremamente prematuros, muito prematuros e prematuros moderados a tardio, os resultados não tiveram relevância estatística.

Ao correlacionar a quantidade de segmentos consonantais e vocálicos à quantidade de processos fonológicos com participantes de extremo baixo peso, muito baixo peso e baixo peso, os achados não foram significativos.

Este estudo apresentou alguns diferenciais para a investigação da aquisição da linguagem: estudo longitudinal com amostras de fala espontânea, que confrontou os dados de 11 crianças divididas em três grupos de idade gestacionais, em três grupos de pesos. Além disso, realizou-se análise estatística de regressão multivariável, controlando participantes e coletas. A ausência de controle dos "participantes" e das "coletas" poderia superestimar o efeito, ou seja, mostrar relevância entre um grupo de participantes ou em determinadas coletas em detrimento de outras.

De forma geral, observou-se a variabilidade dos participantes quanto aos fatores extralinguísticos peso, idade gestacional e idade corrigida. A idade corrigida apresentou relevância na análise estatística quanto à aquisição prosódica e segmental, mostrando a presença do atraso linguístico. Além disso, os prematuros da presente pesquisa ainda estavam em aquisição do inventário segmental, da estrutura silábica e da palavra fonológica.

Os achados deste estudo quanto à existência de atraso em prematuros, independente do grau de prematuridade e da estratificação de peso, possuem implicações clínicas, educacionais e sociais, pois todos precisam ter conhecimento do risco de déficit linguístico em prematuros. 
Vale ressaltar a importância do acompanhamento do desenvolvimento global dos prematuros, de forma longitudinal, para que a presença do atraso seja identificada precocemente, evitando que se perdure, comprometendo o desenvolvimento linguístico ao longo da vida.

Reitera-se a necessidade de mais estudos que incluam também a aquisição segmental e prosódica de crianças prematuras, além das escalas, dos testes e dos questionários avaliativos sobre o desenvolvimento de crianças.

\section{REFERÊNCIAS}

ADAMS-CHAPMAN, I. et al. Neurodevelopmental Impairment Among Extremely Preterm Infants in the Neonatal Research Network. Pediatrics, v. 141, n. 5, e20173091, 2018. Disponível em: https://pediatrics.aappublications.org/content/141/5/e20173091. Acesso em: 13 abr. 2020.

AGARWAL, P. K. et al. Factors affecting neurodevelopmental outcome at 2 years in very preterm infants below 1250 grams: a prospective study. Journal of Perinatology, v. 38, n. 8, p. 1093-1100, aug. 2018. DOI 10.1038/s41372-018-0138-3.

CHEONG, J. L. et al. Association Between Moderate and Late Preterm Birth and Neurodevelopment and Social-Emotional Development at Age 2 Years. JAMA Pediatrics, v. 171, n. 4, e164805, apr. 2017a. Disponível em: https://jamanetwork.com/journals/jamapediatrics/fullarticle/2600001. Acesso em: 02 mai. 2020.

CHEONG, J. L. et al. Brain Volumes at Term-Equivalent Age Are Associated with 2-Year Neurodevelopment in Moderate and Late Preterm Children. The Journal of Pediatrics, v. 171, n. 4, e164805, 2017b.

CLEMENTS, G. N. The Role of Features in Phonological Inventories. In: CLEMENTS, G. N. Laboratoire de Phonétique et Phonologie (UMR 7018). Paris: CNRS / Sorbonne-Nouvelle, 2005. cap 1, p. 1-57.

ELEUTÉRIO, F. J. C. et al.Protocolos de obstetrícia da Secretaria da Saúde do Estado do Ceará.Fortaleza: Secretaria da Saúde do Estado do Ceará, p. 536, 2014. Disponível em: https://www.saude.ce.gov.br/wp-

content/uploads/sites/9/2018/06/protocolos_obstetricia_sesa_ce_2014_.pdf. Acesso em: 03 de jun. 2021.

FELIX, J.; SANTOS, M. E.; BENITEZ-BURRACO, A. Linguagem espontânea de crianças prematuras aos 4 e 5 anos de idade. Revista CEFAC, São Paulo, v. 19, n. 6, p. 742-748, dez. 2017. Disponível em: http://www.scielo.br/scielo.php?script=sci_arttext\&pid=S151618462017000600742\&lng=en\&nrm=iso. Acesso em: 02 mai. 2020.

FORMIGA, C. K. M. R.; VIEIRA, M. E. B.; LINHARES, M. B. M. Avaliação do desenvolvimento de bebês nascidos pré-termo: a comparação entre idades cronológica e corrigida. Revista Brasileira de Crescimento e Desenvolvimento Humano, São Paulo, v. 25, n. 2, p. 230-236, 2015. Disponível em: http://pepsic.bvsalud.org/pdf/rbcdh/v25n2/pt_15.pdf. Acesso em: 26 out. 2019. 
FREITAS, G. C. M. Sobre a aquisição das plosivas e nasais. In: LAMPRECHT, R. R. (org.). Aquisição fonológica do português: perfil de desenvolvimento e subsídios para terapia. Porto Alegre: ArtMed, 2004. p. 73-81..

HOLLANDERS, J. J. et al. Long-Term Neurodevelopmental and Functional Outcomes of Infants Born Very Preterm and/or with a Very Low Birth Weight. Neonatologia, v. 115, n. 4, p. 310-319, 2019. Disponível em: https://www.karger.com/Article/FullText/495133. Acesso em: 01 mai. 2020.

IONIO, C. et al. Paths of cognitive and language development in healthy preterm infants. Infant Behavior and Development, v. 44, p. 199-207, aug. 2016.

JOHNSON, S. et al. Neurodevelopmental outcomes following late and moderate prematurity: a population-based cohort study. Archives of Disease in Childhood: Fetal \& Neonatal, v. 100, n. 4, p.301-308, 2015. Disponível em: https://fn.bmj.com/content/fetalneonatal/100/4/F301.full.pdf. Acesso em: 13 abr. 2020.

LAMPRECHT, R. R. et al. Aquisição fonológica do português: Perfil de desenvolvimento e subsídios para terapia. Porto Alegre: ArtMed, 2004.

MATZENAUER, C. L. B. Gramática(s) fonológica(s) no processo de aquisição da linguagem: a construção do inventário segmental. Prolíngua, João Pessoa, v. 10, n. 1 p. $\quad$ 62-78, 2015. Disponível em: https://periodicos.ufpb.br/index.php/prolingua/article/view/27588/14827. Acesso em: 25 jun. 2017.

MATZENAUER, C. L. B.; MIRANDA, A. R. M. Traços distintivos e a aquisição das vogais do PB. In: HORA, D. da. (org.). Vogais no Ponto mais Oriental das Américas. João Pessoa: Ideia, 2009. p. 45-63.

MEZZOMO, C. L.; RIBAS, L. P. Sobre a Aquisição das líquidas. In: LAMPRECHT, R. R. (org.). Aquisição fonológica do português: perfil de desenvolvimento e subsídios para terapia. Porto Alegre: ArtMed, 2004. p. 95-109.

MIRANDA, A. R. M.; MATZENAUER, C. L. B. Aquisição da fala e da escrita: relações com a Fonologia. Cadernos de Educação, Pelotas, n. 35, p. 359-40, 2010. Disponível em: https://periodicos.ufpel.edu.br/ojs2/index.php/caduc/article/view/1626/1509. Acesso em: 8 dez. 2018.

MONTEIRO-LUPERI, T. I. et al. Desempenho linguístico de prematuros de 2 anos, considerando idade cronológica e idade corrigida. CoDAS, São Paulo, v. 28, n. 2, p. 118-122, 2016. Disponível em: https://www.scielo.br/pdf/codas/v28n2/2317-1782-codas-282-118.pdf. Acesso em: 26 out. 2019.

MOTA, H. B. Aquisição segmental do português: um modelo implicacional de complexidade de traços. 1996. Tese (Doutorado em Letras) - Pontifícia Universidade Católica do Rio Grande do Sul, Porto Alegre,1996. 
OLIVEIRA, C. C. Sobre a aquisição das fricativas. In: LAMPRECHT, R. R. (org.). Aquisição fonológica do português: perfil de desenvolvimento e subsídios para terapia. Porto Alegre: ArtMed, 2004. p. 83-94.

ORGANIZAÇÃO MUNDIAL DA SAÚDE. Survive and thrive: transforming care for every small and sick newborn. Geneva: World Health Organization, 2019. Disponível em: https://apps.who.int/iris/bitstream/handle/10665/326495/9789241515887-eng.pdf?ua=1.

Acesso em: 02 mai. 2020.

PAYÃO, L. M. C. Aquisição de fonologia: a influência do acento e o preenchimento de unidades prosódicas em dados de fala de duas crianças entre 1;0.4 e 2;1.10 de idade, em contato com o português brasileiro falado em Alagoas e Pernambuco. 2010. 192 f. Tese (Doutorado em Linguística) - Faculdade de Letras, Universidade Federal de Alagoas, Maceió, 2010.

PAYÃO, L. M. C.; COSTA, J. F. Preenchimento de unidades prosódicas na aquisição fonológica inicial do português brasileiro. Letras de Hoje, Porto Alegre, v. 51, n. 3, p. 433-441, jul./set. 2016.

PITTET-METRAILLER, M. P. et al. Neurodevelopmental outcome at early school age in a Swiss national cohort of very preterm children. Swiss Medical Weekly, v. 149, w20084, 2019. Disponível em: https://smw.ch/article/doi/smw.2019.20084. Acesso em: 02 mai. 2020.

PUTNICK, D. L. et al. Long-Term Stability of Language Performance in Very Preterm, ModerateLate Preterm, and Term Children. The Journal of Pediatrics, v. 181, p. 74-79, feb. 2017. Disponível em: https://www.ncbi.nlm.nih.gov/pmc/articles/PMC5274586/. Acesso em: 26 out. 2019.

RIBAS, L. P. Sobre a Aquisição do Onset Complexo. In: LAMPRECHT, R. R. (org.). Aquisição fonológica do português: perfil de desenvolvimento e subsídios para terapia. Porto Alegre: ArtMed, 2004. p. 83-94.

RIBEIRO, C. da C. et al. Receptive language and intellectual abilities in preterm children. Early Human Development, v. 99, p. 57-60, 2016. Disponível em: file:// C:/Users/user/Downloads/Receptivelanguageandintellectualabilitiesinpretermchildren.p df. Acesso em: 02 mai. 2020.

SANCHEZ, K. et al. Language in 2-year-old children born preterm and term: a cohort study. Archives of Disease in Childhood, v.104, p. 647-652, 2019.

SILVA, K. F. T.; PAYÃO, L. M. C. Aspectos fonológicos em crianças pré-termo, na faixa etária de 12 a 25 meses. Cadernos de Linguística, Campinas, v. 1, n. 2, p. 1-18, set. 2020. Disponível em: https://cadernos.abralin.org/index.php/cadernos/article/view/209/110. Acesso em: 20 set. 2020.

SOARES, A. C. C.; SILVA, K.; ZUANETTI, P. A. Variáveis de risco para o desenvolvimento da linguagem associadas à prematuridade. Audiology, Communication Research, São Paulo, v. 22, e1745, 2017. Disponível em: https://www.scielo.br/pdf/acr/v22/2317-6431-acr-2317-64312016-1745.pdf. Acesso em: 13 abr. 2020.

WIETHAN, F. M.; MOTA, H. B.; MORAES, A. B. Correlações entre a produção de classes fonêmicas e classes gramaticais no Português Brasileiro. Audiology - Communication Research. 
v. 21, n. 0, e1669, 2016. Disponível em: https://doi.org/10.1590/2317-6431-2015-1669. Acesso em: 03 de jun. 2021.

WERTZNER, H. F. Articulação: aquisição do sistema fonológico dos 3 aos 7 anos. 1992. Tese (Doutorado em Linguística) - Faculdade de Filosofia, Letras e Ciências Humanas, Universidade de São Paulo, São Paulo, 1992.

ZAGO, J. T. C. et al. Associação entre o desenvolvimento neuropsicomotor e fatores de risco biológico e ambientais em crianças na primeira infância. Revista CEFAC, São Paulo, v. 19, n. 3, p. 320-329, 2017. Disponível em: https://www.scielo.br/pdf/rcefac/v19n3/1982-0216-rcefac-1903-00320.pdf. Acesso em: 13 abr. 2020.

ZERBETO, A. B.; CORTELO, F. M.; FILHA, E. B. C. Association between gestational age and birth weight on the language development of brazilian children: a systematic review. Jornal de Pediatria (Rio J.), Porto Alegre, v. 91, p. 326-332, 2015. Disponível em:

https://www.scielo.br/pdf/jped/v91n4/pt_0021-7557-jped-91-04-00326.pdf. Acesso em: 26 out. 2019. 